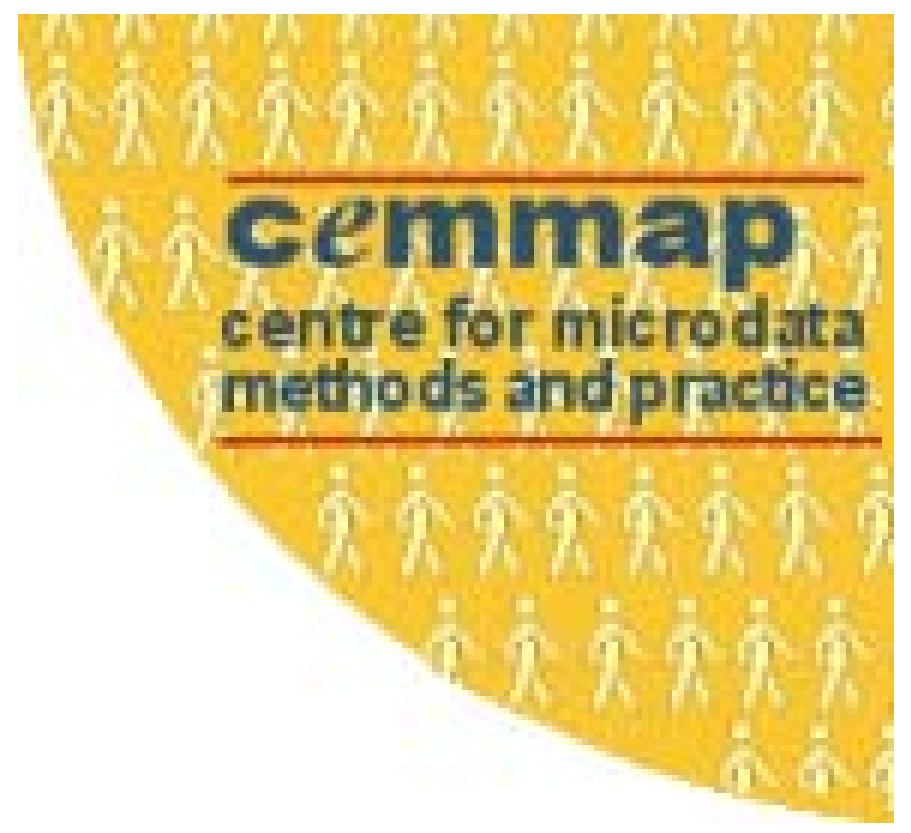

NONPARAMETRIC IDENTIFICATION WITH DISCRETE ENDOGENOUS VARIABLES

Andrew Chesher

THE INSTITUTE FOR FISCAL STUDIES DEPARTMENT OF ECONOMICS, UCL cemmap working paper CWP06/03 


\title{
Nonparametric identification with discrete endogenous variables
}

\author{
AndRew Chesher* \\ Centre for Microdata Methods and Practice \\ Institute for Fiscal Studies and University College London
}

July 29th 2003, revised September 8th $2003^{\dagger}$

\begin{abstract}
This paper studies the nonparametric identification of partial differences of a nonseparable structural function that determines the value of a discrete or continuous random variable when the function depends on endogenous variates which have discrete probability distributions. Weak conditions are developed under which a partial difference of a structural function with respect to a discrete endogenous variable is nonparametrically identified up to an interval. The interval is short when there are many points of support of the endogenous variable. The interval has finite length if there are at least three points of support but not when there are only two. The interval can be estimated using quantile regression estimation methods.
\end{abstract}

\section{INTRODUCTION}

1.1. Nonseparable models and identification of partial differences. This paper considers the identification of a partial difference of a nonseparable structural function that yields the value of a discrete or continuous random variable, a function which includes among its arguments endogenous variates which have discrete probability distributions.

Weak conditions are developed under which a partial difference of a structural function with respect to a discrete endogenous variable is nonparametrically identified to within an interval. The interval can be estimated using quantile regression estimation procedures.

As the granularity of the support of a discrete endogenous variable diminshes, the interval shrinks to a point, yielding a point identification result when the endogenous variable is continuously distributed.

To motivate the study of this problem consider the following stylised Becker-ChiswickMincer model ${ }^{1}$ of the determination of the log wage $(W)$ and completed years of schooling $(S)$.

$$
\begin{aligned}
W & =h_{1}\left(S, X, \rho_{1}\right) \\
S & =h_{2}\left(X, \rho_{2}\right)
\end{aligned}
$$

Here $X$ denotes a list of covariates measuring characteristics of the individual and of the environment in which decisions are made and outcomes determined, and $\rho_{1}$ and $\rho_{2}$ are unobserved, continuously, and possibly dependently distributed random variables. The variate $\rho_{2}$ will be interpreted as a measure of ability.

\footnotetext{
*I am grateful to Roger Koenker and to seminar participants at cemmap, Northwestern University, University of Chicago and at the Harvard-MIT econometrics workshop for comments on the related paper Chesher (2002) and for discussion of the problem considered in this paper.

†This revision corrects errors in the presentation of the proof of Lemma 2 in which, in the July 29th version, some inequalities were incorrectly oriented.

${ }^{1}$ See Becker and Chiswick (1966), Chiswick and Mincer (1972), Chiswick (1974), Mincer (1974), Card (1995), Card (2001)
} 
In this context there is interest in partial differences like

$$
\Delta \equiv h_{1}\left(s^{\prime}, x, r_{1}\right)-h_{1}\left(s^{\prime \prime}, x, r_{1}\right)
$$

which measure the "pure" effect of schooling on the wage.

When $\rho_{1}$ and $\rho_{2}$ are dependently distributed these "pure" effects cannot be identified without adding further restrictions to the model and $(W, S, X)$ data can only reveal the combined effect on the wage of schooling (via the $S$-argument of $h_{1}$ ) and ability (via the $\rho_{1}$-argument of $h_{1}$ through its dependence on $\rho_{2}$ ).

Chesher (2003) develops weak conditions under which partial derivatives of structural functions like $h_{1}$ are nonparametrically point identified, but those results are not applicable to the problem studied here because, when $S$ is a discrete random variable, there cannot be nonparametric identification of the $S$-partial derivative of the function $h_{1}$. That paper also surveys the related literature.

Weak conditions under which there is nonparametric point identification of a partial difference, like $\Delta$, when endogenous variables ( $S$ above) are continuously distributed but covariates vary only discretely are developed in Chesher (2002). Discrete variation in $X$ prohibits nonparametric identification of the $S$-partial derivative of the function $h_{1}$, hence the focus in that paper on identification of partial differences.

The method employed in Chesher (2002) is not directly applicable when $S$ has a discrete distribution. The discrete endogenous variable case was left in that paper as a puzzle which this paper solves by developing weak conditions under which an interval containing a structural partial difference like $\Delta$ can be nonparametrically identified.

1.2. Interval identification of a partial difference. The interval identification result is now introduced in the context of a simple restricted version of the model at the start of this Section in which the covariates, $X$, are excluded from the wage structural function, $h_{1}$, as follows.

$$
\begin{aligned}
W & =h_{1}\left(S, \rho_{1}\right) \\
S & =h_{2}\left(X, \rho_{2}\right)
\end{aligned}
$$

Let $\left\{s_{m}\right\}_{m=1}^{M}$ be the points of support of the discrete distribution of $S$ and consider two distinct points of support, $s_{i}$ and $s_{j}$ and the neighbouring points of support below them, $s_{i-1}$ and $s_{j-1}$.

The variate $\rho_{2}$ is normalised to be uniformly distributed on $(0,1)$ independent of $X$. The function $h_{2}\left(X, \rho_{2}\right)$ is therefore the conditional quantile function of $S$ given $X$, a weakly increasing caglad step function with steps whose lengths are equal to the probability masses on the points of support of the distribution of $S$ given $X$.

Let $r_{1}$ be a value of continuously distributed $\rho_{1}$, to be specified. Identification of the structural partial difference

$$
\Delta \equiv h_{1}\left(s_{j}, r_{1}\right)-h_{1}\left(s_{i}, r_{1}\right)
$$

is considered.

Sufficient conditions for identification of $\Delta$ are as follows. ${ }^{2}$

1. Monotonicity. The function $h_{1}$ is strictly monotonically varying with $\rho_{1}$. The conditional $\tau_{1}$-quantile of $\rho_{1}$ given $\rho_{2}$ and $X$ is weakly monotonically varying with $\rho_{2} \cdot{ }^{3}$

\footnotetext{
${ }^{2}$ Weaker conditions are set down in Section 2.

${ }^{3}$ The second monotonicity condition is only required to hold over intervals of values of $\rho_{2}$.
} 
2. Rank condition. There exist four distinct values of $X: x^{i}, x^{i-1}, x^{j}$ and $x^{j-1}$ such that for a chosen value $\tau_{2} \in(0,1)$

$$
\tau_{2}=P\left[S=s^{i} \mid x^{i}\right]=P\left[S=s^{i-1} \mid x^{i-1}\right]=P\left[S=s^{j} \mid x^{j}\right]=P\left[S=s^{j-1} \mid x^{j-1}\right] .
$$

These covariate values are referred to as instrumental values. ${ }^{4}$

3. Covariation. The value of the $\tau_{1}$-quantile of $\rho_{1}$ given $\rho_{2}=\tau_{2}$ (see equation (2)) and $X$ does not vary across the four instrumental values. Denote the common value by $r_{1}$ - this is the value of $\rho_{1}$ that appears in the definition of the partial difference $\Delta$, given in equation (1). ${ }^{5}$

The interval identification result makes use of conditional quantile functions. The conditional $\tau$-quantile of a random variable $A$ given a (possibly vector) random variable $B=b$ is denoted by $Q_{A \mid B}(\tau \mid b),{ }^{6}$ defined as

$$
Q_{A \mid B}(\tau \mid b) \equiv \inf \left\{q: F_{A \mid B}(q \mid b) \geq \tau\right\}
$$

where $F_{A \mid B}$ is the conditional distribution function of $A$ given $B$.

Under the conditions just stated there is the following inequality

$$
\begin{gathered}
\min \left(\begin{array}{c}
Q_{W \mid S X}\left(\tau_{1} \mid s_{j}, x^{j}\right)-Q_{W \mid S X}\left(\tau_{1} \mid s_{i}, x^{i-1}\right), \\
Q_{W \mid S X}\left(\tau_{1} \mid s_{j}, x^{j-1}\right)-Q_{W \mid S X}\left(\tau_{1} \mid s_{i}, x^{i}\right)
\end{array}\right) \\
\leq \Delta \leq \\
\max \left(\begin{array}{c}
Q_{W \mid S X}\left(\tau_{1} \mid s_{j}, x^{j}\right)-Q_{W \mid S X}\left(\tau_{1} \mid s_{i}, x^{i-1}\right), \\
Q_{W \mid S X}\left(\tau_{1} \mid s_{j}, x^{j-1}\right)-Q_{W \mid S X}\left(\tau_{1} \mid s_{i}, x^{i}\right)
\end{array}\right)
\end{gathered}
$$

where the values of $S$ that appear in (3) are conditional $\tau_{2}$-quantiles of the distribution of $S$ given $X$, as follows. ${ }^{7}$

$$
\left.\begin{array}{rl}
s_{i}=Q_{S \mid X}\left(\tau_{2} \mid x^{i}\right) & s_{i-1}=Q_{S \mid X}\left(\tau_{2} \mid x^{i-1}\right) \\
s_{j}=Q_{S \mid X}\left(\tau_{2} \mid x^{j}\right) & s_{j-1}=Q_{S \mid X}\left(\tau_{2} \mid x^{j-1}\right)
\end{array}\right\}
$$

This inequality identifies $\Delta$ up to an interval which can be estimated via the analogue principle (Manski (1988)), replacing the conditional quantiles in (3) by estimated conditional quantiles evaluated at estimates of the instrumental values of $X$. The inequality (3) holds when $W$ is discretely or continuously distributed.

This Section concludes with a discussion of some issues raised by the result (3). Section 2 gives a formal statement of the identifying restrictions and a Theorem stating the interval identification result.

1.3. Estimation and overidentification. To estimate the bounding interval calculate an estimate $\hat{F}_{S \mid X}$ and then find values, $\left\{\hat{x}^{i}, \hat{x}^{i-1}, \hat{x}^{j}, \hat{x}^{j-1}\right\}$ of $X$ such that

$$
\tau_{2}=\hat{F}_{S \mid X}\left(s_{i} \mid \hat{x}^{i}\right)=\hat{F}_{S \mid X}\left(s_{i-1} \mid \hat{x}^{i-1}\right)=\hat{F}_{S \mid X}\left(s_{j} \mid \hat{x}^{j}\right)=\hat{F}_{S \mid X}\left(s_{j-1} \mid \hat{x}^{j-1}\right) .
$$

\footnotetext{
${ }^{4}$ See Chesher (2002). Note that the existence of such instrumental values for any chosen $\tau_{2}$ will depend on the nature and extent of the support of $X$.

${ }^{5}$ This would of course be satisfied if $\rho_{1}$ were distributed independently of $X$ given $\rho_{2}$, but is far less restrictive than an independence condition being specific to $\tau_{1}, \tau_{2}$ and the values of $X$ considered.

${ }^{6}$ For example $Q_{A \mid B_{1} B_{2}}\left(\tau \mid b_{1}, b_{2}\right)$ is the $\tau$-quantile of $A$ given $B_{1}=b_{1}$ and $B_{2}=b_{2}$.

${ }^{7}$ Since $S$ has a discrete distribution these values of $S$ are also conditional $\tau$-quantiles for some values of $\tau \neq \tau_{2}$.
} 
Finally calculate estimates, $\hat{Q}_{W \mid S X}\left(\tau_{1} \mid s_{i}, x\right)$ for $x \in\left\{\hat{x}^{i}, \hat{x}^{i-1}\right\}$ and $\hat{Q}_{W \mid S X}\left(\tau_{1} \mid s_{j}, x\right)$ for $x \in\left\{\hat{x}^{j}, \hat{x}^{j-1}\right\}$ and substitute in (3). At each stage the estimates could be calculated using a parametric, semi- or nonparametric estimator. ${ }^{8}$

When $X$ contains many covariates there may be many choices of instrumental values which yield the same identifying interval. Then the interval is overidentified and the procedure described above will yield many estimates of the identifying interval. One could perhaps combine these using a minimum distance procedure to produce an asymptotically efficient estimator.

In the just identified case, if $F_{S \mid X}$ does not vary monotonically with $x$ there may be distinct sets of instrumental values leading to different identifying intervals. One would naturally be lead to use the set of instrumental values yielding the shortest interval although accuracy of estimation will be a factor to bear in mind when choosing among sets of instrumental values.

1.4. Rich support. Suppose that the granularity of the support of $S$ is very fine, so that $S$ is "nearly" continuously distributed. Then if $F_{S \mid X}(s \mid x)$ is smoothly varying with $x$ it will be possible to choose $x^{i-1}$ close to $x^{i}$ and $x^{j-1}$ close to $x^{j}$ and then, if $Q_{\rho_{1} \mid \rho_{2} X}$ is smoothly varying with $\rho_{2}$, the separation between the bounds on $\Delta$ will be small.

If in fact $S$ is continuously distributed then, if there exist instrumental values $x^{i}$ and $x^{j}$, one can choose $x^{i-1}=x^{i}$ and $x^{j-1}=x^{j}$ and the inequality (3) collapses, yielding the point identification result given in Chesher (2002)

$$
\Delta=Q_{W \mid S X}\left(\tau_{1} \mid s_{j}, x^{j}\right)-Q_{W \mid S X}\left(\tau_{1} \mid s_{i}, x^{i}\right)
$$

where

$$
\begin{aligned}
& s_{i}=Q_{S \mid X}\left(\tau_{2} \mid x^{i}\right) \\
& s_{j}=Q_{S \mid X}\left(\tau_{2} \mid x^{j}\right) .
\end{aligned}
$$

When $S$ is continuously distributed, $\tau_{2}$ is the unique probability value which satisfies these equations.

1.5. Discrete covariates. If $X$ has limited variation, for example when $X$ is discrete, it may not be possible to find any instrumental values that satisfy (2) for a particular, or perhaps for any choice of $s_{i}$ and $s_{j}$ at any value of $\tau_{2}$. It is sometimes possible to develop wider identifying intervals in this case as explained in Section 3.3.

1.6. Binary endogenous variables. If $j=i \pm 1$ the interval identifying inequality (3) involves three points of support of the discrete endogenous variable, otherwise it involves four points of support. Suppose the endogenous variable has just two points of support, without loss of generality $\{0,1\}$. Then (3) does not apply in full. However, following the argument in the Appendix, it can be shown that if the conditional $\tau_{1}$-quantile of $\rho_{1}$ given $\rho_{2}$ and $X$ is non-decreasing with $\rho_{2}$ there is the inequality:

$$
\Delta \geq Q_{W \mid S X}\left(\tau_{1} \mid 1, x^{0}\right)-Q_{W \mid S X}\left(\tau_{1} \mid 0, x^{0}\right)
$$

with the inequality reversed if the conditional $\tau_{1}$ quantile of $\rho_{1}$ given $\rho_{2}$ and $X$ is nonincreasing with $\rho_{2}$.

So, in the binary endogenous variable case, any knowledge of $\Delta$ under the weak conditions set out here requires prior knowledge of the direction of the dependence of $\rho_{1}$ on

\footnotetext{
${ }^{8}$ Regarding the quantile function estimation: for parametric estimation, see Koenker and Bassett (1978) and Koenker and d'Orey (1987); for semiparametric estimation see Chaudhuri, Doksum and Samarov (1997), Kahn (2001) and Lee (2003); for nonparametric estimation, see Chaudhuri (1991).
} 
$\rho_{2}$. Even with that knowledge, $\Delta$ can only be nonparametrically identified in this nonseparable model up to an interval with infinite length. More informative identification in the presence of binary endogenous variables requires stronger restrictions than those considered here. ${ }^{9}$

1.7. Plan of the remainder of the paper. The next Section provides a full set of identifying restrictions, rather weaker than those used in introducing the result and a Theorem stating an interval identification result for a structural partial difference with respect to an endogenous variate for the case in which the structural function depends on covariates and just one endogenous variate. The proof is contained in the Appendix.

Section 3 considers three extensions: interval identification when the structural function depends on more than one endogenous variate; interval identification of a structural partial difference with respect to a covariate; and interval identification when covariates have sparse support insufficient to allow the existence of instrumental values of $X$ satisfying all the equalities in condition (2). The final Section concludes.

\section{INTERVAL IDENTIFICATION OF STRUCTURAL PARTIAL DIFFERENCES}

Consider a model for two outcomes, $Y_{1}$ and $Y_{2}$ with covariates $X=\left\{X_{k}\right\}_{k=1}^{K}$ and two continuously distributed latent random variables $\rho_{1}$ and $\rho_{2}$ with structural equations as follows.

$$
\begin{aligned}
& Y_{1}=h_{1}\left(Y_{2}, X, \rho_{1}\right) \\
& Y_{2}=h_{2}\left(X, \rho_{2}\right)
\end{aligned}
$$

The conditional distribution function of $\rho_{1}$ given $\rho_{2}$ and $X$ is denoted by $F_{\rho_{1} \mid \rho_{2} X}$ and $Q_{\rho_{1} \mid \rho_{2} X}$ denotes the associated conditional quantile function. The distribution of $\rho_{2}$ is normalised as uniform on $(0,1)$ independent of $X$.

2.1. The discrete distribution of the endogenous variable. Let the points of support of $Y_{2}$ be $\left\{y_{2}^{m}\right\}_{m=1}^{M}$, independent of $X$, with $M$ not necessarily finite. The associated positive probability masses are $\left\{p_{m}(x)\right\}_{m=1}^{M}$ with $\sum_{m=1}^{M} p_{m}(x)=1$. Define the cumulative probability sums $\left\{p^{m}(x)\right\}_{m=1}^{M}$ as follows.

$$
p^{m}(x) \equiv \sum_{n=1}^{m} p_{n}(x), \quad m \in\{1, \ldots, M\}
$$

The distribution and quantile functions of $Y_{2}$ given $X=x$ are as follows.

$$
\begin{gathered}
F_{Y_{2} \mid X}\left(y_{2} \mid x\right)=\left\{\begin{array}{cl}
0 & -\infty<y_{2}<y_{2}^{1} \\
p^{m}(x) & y_{2}^{m} \leq y_{2}<y_{2}^{m+1} \\
1 & y_{2}^{M} \leq y_{2}<\infty
\end{array} \quad m \in\{2, \ldots, M-1\}\right. \\
Q_{Y_{2} \mid X}(\tau \mid x)=\left\{\begin{array}{cl}
y_{2}^{1} & 0<\tau \leq p^{1}(x) \\
y_{2}^{m} & p^{m-1}(x)<\tau \leq p^{m}(x), \\
y_{2}^{M} & p^{m}(x)<\tau \leq 1
\end{array}\right.
\end{gathered}
$$

Since $\rho_{2}$ is normalised as uniformly distributed on $(0,1)$ independent of $X$, the structural function $h_{2}$ is the quantile function of $Y_{2}$ given $X$, that is:

$$
h_{2}\left(x, \rho_{2}\right)=Q_{Y_{2} \mid X}\left(\rho_{2} \mid x\right) .
$$

\footnotetext{
${ }^{9}$ For example parametric restrictions.
} 
2.2. Interval identifying restrictions and a Theorem. Consider probabilities $\left\{\tau_{1}, \tau_{2}\right\} \in(0,1)^{2}$ and two points of support of $Y_{2}, y_{2}^{i} \neq y_{2}^{j}$. There are the following conditions.

I. Triangularity. At any value of $X$, and of continuously distributed $\rho_{1}$ and $\rho_{2}$, the values of the outcomes $Y_{1}$ and $Y_{2}$ are uniquely determined by equations (5) and (6).

II. Rank condition. There exists a set of instrumental values of $X$ :

$$
\mathcal{X}=\left\{x^{i}, x^{j}, x^{i-1}, x^{j-1}\right\}
$$

such that

$$
p^{i}\left(x^{i}\right)=p^{j}\left(x^{j}\right)=p^{i-1}\left(x^{i-1}\right)=p^{j-1}\left(x^{j-1}\right)=\tau_{2} .
$$

III. Monotonicity. The function $h_{1}$ is weakly monotonic with respect to variation in $\rho_{1}$, normalised non-decreasing and caglad. For $x \in \mathcal{X}$, and

$$
r_{2} \in \mathcal{R}_{2}(x) \equiv\left[p^{i-1}(x), p^{i}(x)\right] \cup\left[p^{j-1}(x), p^{j}(x)\right]
$$

the conditional quantile function $Q_{\rho_{1} \mid \rho_{2} X}\left(\tau_{1} \mid r_{2}, x\right)$ is either a non-increasing or nondecreasing function of $r_{2} \cdot{ }^{10}$

IV. Covariation. For $\left\{x^{\prime}, x^{\prime \prime}\right\} \in \mathcal{X}$ and $r_{2} \in \mathcal{R}_{2}\left(x^{\prime}\right) \cup \mathcal{R}_{2}\left(x^{\prime \prime}\right)$,

$$
Q_{\rho_{1} \mid \rho_{2} X}\left(\tau_{1} \mid r_{2}, x^{\prime}\right)=Q_{\rho_{1} \mid \rho_{2} X}\left(\tau_{1} \mid r_{2}, x^{\prime \prime}\right)
$$

and $\rho_{2}$ is normalised to be uniformly distributed on $(0,1)$ independent of $X$.

V. Order condition. For $x \in \mathcal{X}$, define $r_{1} \equiv Q_{\rho_{1} \mid \rho_{2} X}\left(\tau_{1} \mid \tau_{2}, x\right) .{ }^{11}$ For $\left\{x^{\prime}, x^{\prime \prime}\right\} \in \mathcal{X}$ and $l \in\{i, i-1, j, j-1\}$

$$
h_{1}\left(y_{2}^{l}, x^{\prime}, r_{1}\right)=h_{1}\left(y_{2}^{l}, x^{\prime \prime}, r_{1}\right)
$$

For $x \in \mathcal{X}$ define the following structural partial difference.

$$
\Delta_{\mathcal{X}}\left(y_{2}^{i}, y_{2}^{j}, \tau_{1}, \tau_{2}\right) \equiv h_{1}\left(y_{2}^{j}, x, r_{1}\right)-h_{1}\left(y_{2}^{i}, x, r_{1}\right)
$$

The Theorem stating the set identification of $\Delta_{\mathcal{X}}\left(y_{2}^{i}, y_{2}^{j}, \tau_{1}, \tau_{2}\right)$ is as follows.

Theorem 1. Conditions I - V imply the following inequality.

$$
\begin{gathered}
\min \left(\begin{array}{c}
Q_{Y_{1} \mid Y_{2} X}\left(\tau_{1} \mid y_{2}^{j}, x^{j}\right)-Q_{Y_{1} \mid Y_{2} X}\left(\tau_{1} \mid y_{2}^{i}, x^{i-1}\right), \\
Q_{Y_{1} \mid Y_{2} X}\left(\tau_{1} \mid y_{2}^{j}, x^{j-1}\right)-Q_{Y_{1} \mid Y_{2} X}\left(\tau_{1} \mid y_{2}^{i}, x^{i}\right)
\end{array}\right) \\
\leq \Delta_{\mathcal{X}}\left(y_{2}^{i}, y_{2}^{j}, \tau_{1}, \tau_{2}\right) \leq \\
\max \left(\begin{array}{c}
Q_{Y_{1} \mid Y_{2} X}\left(\tau_{1} \mid y_{2}^{j}, x^{j}\right)-Q_{Y_{1} \mid Y_{2} X}\left(\tau_{1} \mid y_{2}^{i}, x^{i-1}\right), \\
Q_{Y_{1} \mid Y_{2} X}\left(\tau_{1} \mid y_{2}^{j}, x^{j-1}\right)-Q_{Y_{1} \mid Y_{2} X}\left(\tau_{1} \mid y_{2}^{i}, x^{i}\right)
\end{array}\right)
\end{gathered}
$$

The Theorem is proved in the Appendix. The method of proof is as follows.

\footnotetext{
${ }^{10}$ This is somewhat stronger then is required but has the virtue of simplicity - see the Appendix for discussion.

${ }^{11}$ Note $r_{1}$ is invariant with respect to $x \in \mathcal{X}$ by virtue of Condition IV.
} 
2.3. The method of proof. First an expression is obtained for the conditional distribution function of $Y_{1}$ given $Y_{2}=y_{2}^{m}$ (one of the points of support) and $X=x$. This leads directly to an equation whose solution is the conditional quantile function of $Y_{1}$ given $Y_{2}=y_{2}^{m}$ and $X=x$.

When $F_{\rho_{1} \mid \rho_{2} X}$ is non-decreasing in $\rho_{2}$ for $\rho_{2} \in\left[p^{m-1}(x), p^{m}(x)\right]$ the solution can be bounded, as follows:

$h_{1}\left(y_{2}^{m}, x, Q_{\rho_{1} \mid \rho_{2} X}\left(\tau_{1} \mid p^{m}(x), x\right)\right) \leq Q_{Y_{1} \mid Y_{2} X}\left(\tau_{1} \mid y_{2}^{m}, x\right) \leq h_{1}\left(y_{2}^{m}, x, Q_{\rho_{1} \mid \rho_{2} X}\left(\tau_{1} \mid p^{m-1}(x), x\right)\right)$

with the inequalities reversed when $F_{\rho_{1} \mid \rho_{2} X}$ is non-increasing in $\rho_{2}$ for $\rho_{2} \in\left[p^{m-1}(x), p^{m}(x)\right]$.

In the non-decreasing $F_{\rho_{1} \mid \rho_{2} X}$ case, for $x \in\left\{x^{i}, x^{i-1}\right\}$ with $m$ set equal to $i$ there is, under the conditions of Theorem 1 , from (7)

$$
\begin{aligned}
h_{1}\left(y_{2}^{i}, x, r_{1}\right) & \leq Q_{Y_{1} \mid Y_{2} X}\left(\tau_{1} \mid y_{2}^{i}, x^{i}\right) \\
Q_{Y_{1} \mid Y_{2} X}\left(\tau_{1} \mid y_{2}^{i}, x^{i-1}\right) & \leq h_{1}\left(y_{2}^{i}, x, r_{1}\right)
\end{aligned}
$$

and for $x \in\left\{x^{j}, x^{j-1}\right\}$ with $m$ set equal to $j$

$$
\begin{aligned}
h_{1}\left(y_{2}^{j}, x, r_{1}\right) & \leq Q_{Y_{1} \mid Y_{2} X}\left(\tau_{1} \mid y_{2}^{j}, x^{j}\right) \\
Q_{Y_{1} \mid Y_{2} X}\left(\tau_{1} \mid y_{2}^{j}, x^{j-1}\right) & \leq h_{1}\left(y_{2}^{j}, x, r_{1}\right) .
\end{aligned}
$$

Adding (8) to (11) and rearranging and adding (9) to (10) and rearranging gives

$$
\begin{gathered}
Q_{Y_{1} \mid Y_{2} X}\left(\tau_{1} \mid y_{2}^{j}, x^{j-1}\right)-Q_{Y_{1} \mid Y_{2} X}\left(\tau_{1} \mid y_{2}^{i}, x^{i}\right) \\
\leq h_{1}\left(y_{2}^{j}, x, r_{1}\right)-h_{1}\left(y_{2}^{i}, x, r_{1}\right) \equiv \Delta_{\mathcal{X}}\left(y_{2}^{i}, y_{2}^{j}, \tau_{1}, \tau_{2}\right) \leq \\
Q_{Y_{1} \mid Y_{2} X}\left(\tau_{1} \mid y_{2}^{j}, x^{j}\right)-Q_{Y_{1} \mid Y_{2} X}\left(\tau_{1} \mid y_{2}^{i}, x^{i-1}\right)
\end{gathered}
$$

In the non-increasing $F_{\rho_{1} \mid \rho_{2} X}$ case the inequalities are reversed and the result of Theorem 1 follows on combining the two cases.

\section{Extensions}

3.1. Many endogenous variables. The identification result of Theorem 1 is for the case in which there is a single endogenous variable in the structural function of interest. Extension to the case with more than one endogenous variable is straightforward.

Consider the case in which there are two endogenous variates, $Y_{2}$ and $Y_{3}$ and in place of condition (I), condition $\left(\mathrm{I}^{\prime}\right)$.

$I^{\prime}$. Triangularity. At any value of $X$, and of continuously distributed $\rho_{1}, \rho_{2}$ and $\rho_{3}$, the values of the outcomes $Y_{1}, Y_{2}$ and $Y_{3}$ are uniquely determined by the following equations.

$$
\begin{aligned}
& Y_{1}=h_{1}\left(Y_{2}, Y_{3}, X, \rho_{1}\right) \\
& Y_{2}=h_{2}\left(X, \rho_{2}\right) \\
& Y_{3}=h_{3}\left(X, \rho_{3}\right)
\end{aligned}
$$

Let $y_{3}^{g}$ be a point of support of the distribution of $Y_{3}$ and consider interval identification of the following partial difference with respect to $Y_{2}$

$$
\Delta_{\mathcal{X}}\left(y_{2}^{i}, y_{2}^{j}, y_{3}^{g}, \tau_{1}, \tau_{2}\right) \equiv h_{1}\left(y_{2}^{j}, y_{3}^{g}, x, r_{1}\right)-h_{1}\left(y_{2}^{i}, y_{3}^{g}, x, r_{1}\right)
$$

for $x \in \mathcal{X}$ a set of instrumental values satisfying condition ( $\left.\mathrm{II}^{\prime}\right)$. Now cumulative probabilities are subscripted to indicate the endogenous variate to which they refer. 
II'. Rank condition. There exists a set of instrumental values of $X$ :

$$
\mathcal{X}=\left\{x^{i}, x^{j}, x^{i-1}, x^{j-1}\right\}
$$

such that

$$
\begin{aligned}
& p_{2}^{i}\left(x^{i}\right)=p_{2}^{j}\left(x^{j}\right)=p_{2}^{i-1}\left(x^{i-1}\right)=p_{2}^{j-1}\left(x^{j-1}\right)=\tau_{2} \\
& p_{3}^{g}\left(x^{i}\right)=p_{3}^{g}\left(x^{j}\right)=p_{3}^{g}\left(x^{i-1}\right)=p_{3}^{g}\left(x^{j-1}\right)=\tau_{3} .
\end{aligned}
$$

The monotonicity, covariation and order conditions are amended as follows.

III'. Monotonicity. The function $h_{1}$ is weakly monotonic with respect to variation in $\rho_{1}$, normalised non-decreasing and caglad. For $x \in \mathcal{X}$, and

$$
r_{2} \in \mathcal{R}_{2}(x) \equiv\left[p_{2}^{i-1}(x), p_{2}^{i}(x)\right] \cup\left[p_{2}^{j-1}(x), p_{2}^{j}(x)\right]
$$

the conditional quantile function $Q_{\rho_{1} \mid \rho_{2} \rho_{3} X}\left(\tau_{1} \mid r_{2}, \tau_{3}, x\right)$ is either a non-increasing or non-decreasing function of $r_{2}$.

$I^{\prime}$. Covariation. For $\left\{x^{\prime}, x^{\prime \prime}\right\} \in \mathcal{X}$ and $r_{2} \in \mathcal{R}_{2}\left(x^{\prime}\right) \cup \mathcal{R}_{2}\left(x^{\prime \prime}\right)$,

$$
Q_{\rho_{1} \mid \rho_{2} \rho_{3} X}\left(\tau_{1} \mid r_{2}, \tau_{3}, x^{\prime}\right)=Q_{\rho_{1} \mid \rho_{2} \rho_{3} X}\left(\tau_{1} \mid r_{2}, \tau_{3}, x^{\prime \prime}\right)
$$

and $\rho_{2}$ and $\rho_{3}$ are normalised to be independently uniformly distributed on $(0,1)^{2}$ independent of $X$.

$\mathrm{V}^{\prime}$. Order condition. For $x \in \mathcal{X}$ define $r_{1} \equiv Q_{\rho_{1} \mid \rho_{2} \rho_{3} X}\left(\tau_{1} \mid \tau_{2}, \tau_{3}, x\right)$. For $\left\{x^{\prime}, x^{\prime \prime}\right\} \in \mathcal{X}$ and $l \in\{i, i-1, j, j-1\}$

$$
h_{1}\left(y_{2}^{l}, y_{3}^{g}, x^{\prime}, r_{1}\right)=h_{1}\left(y_{2}^{l}, y_{3}^{g}, x^{\prime \prime}, r_{1}\right) .
$$

Under conditions $\mathrm{I}^{\prime}-\mathrm{V}^{\prime}$ there is the following interval identifying inequality.

$$
\begin{gathered}
\min \left(\begin{array}{c}
Q_{Y_{1} \mid Y_{2} Y_{3} X}\left(\tau_{1} \mid y_{2}^{j}, y_{3}^{g}, x^{j}\right)-Q_{Y_{1} \mid Y_{2} Y_{3} X}\left(\tau_{1} \mid y_{2}^{i}, y_{3}^{g}, x^{i-1}\right), \\
Q_{Y_{1} \mid Y_{2} Y_{3} X}\left(\tau_{1} \mid y_{2}^{j}, y_{3}^{g}, x^{j-1}\right)-Q_{Y_{1} \mid Y_{2} Y_{3} X}\left(\tau_{1} \mid y_{2}^{i}, y_{3}^{g}, x^{i}\right)
\end{array}\right) \\
\leq \Delta_{\mathcal{X}}\left(y_{2}^{i}, y_{2}^{j}, y_{3}^{g}, \tau_{1}, \tau_{2}\right) \leq \\
\max \left(\begin{array}{c}
Q_{Y_{1} \mid Y_{2} Y_{3} X}\left(\tau_{1} \mid y_{2}^{j}, y_{3}^{g}, x^{j}\right)-Q_{Y_{1} \mid Y_{2} Y_{3} X}\left(\tau_{1} \mid y_{2}^{i}, y_{3}^{g}, x^{i-1}\right), \\
Q_{Y_{1} \mid Y_{2} Y_{3} X}\left(\tau_{1} \mid y_{2}^{j}, y_{3}^{g}, x^{j-1}\right)-Q_{Y_{1} \mid Y_{2} Y_{3} X}\left(\tau_{1} \mid y_{2}^{i}, y_{3}^{g}, x^{i}\right)
\end{array}\right)
\end{gathered}
$$

The proof is similar to the proof of the Theorem 1 given in the Appendix.

3.2. Partial differences with respect to covariates. Consider a simple case in which the structural function of interest, $h_{1}$, depends on a single discrete endogenous variable and a single covariate $X \diamond$ with $X=\left(X_{\diamond}, X_{\diamond}\right)$.

Interval identification of the structural partial difference,

$$
\Lambda_{\mathcal{X}}\left(y_{2}^{i}, x^{a}, x^{b}, \tau_{1}, \tau_{2}\right)=h_{1}\left(y_{2}^{i}, x^{a}, r_{1}\right)-h_{1}\left(y_{2}^{i}, x^{b}, r_{1}\right)
$$

requires the existence of four instrumental values, $\mathcal{X}=\left\{x_{a}^{i}, x_{a}^{i-1}, x_{b}^{i}, x_{b}^{i-1}\right\}$ as in condition (II) but with the requirement that within the set of instrumental values, $x \downarrow=x^{a}$ for $x \in\left\{x_{a}^{i}, x_{a}^{i-1}\right\}, x_{\diamond}=x^{b}$ for $x \in\left\{x_{b}^{i}, x_{b}^{i-1}\right\}$ and the probability of obtaining the value, $y_{2}^{i}$, of the endogenous variable given $X=x$ is equal to $\tau_{2}$ for all $x$ within the set of instrumental values. Condition $(\mathrm{V})$ is modified, now requiring that the structural function 
is insensitive, only via its $X_{\diamond}$ arguments, to variation in $x$ within the set of instrumental values. Conditions (I), (III) and (IV) are maintained.

The interval identifying inequality is:

$$
\begin{gathered}
\min \left(Q_{Y_{1} \mid Y_{2} X}\left(\tau_{1} \mid y_{2}^{i}, x_{a}^{i}\right)-Q_{Y_{1} \mid Y_{2} X}\left(\tau_{1} \mid y_{2}^{i}, x_{b}^{i-1}\right), Q_{Y_{1} \mid Y_{2} X}\left(\tau_{1} \mid y_{2}^{i}, x_{a}^{i-1}\right)-Q_{Y_{1} \mid Y_{2} X}\left(\tau_{1} \mid y_{2}^{i}, x_{b}^{i}\right)\right) \\
\leq \Lambda_{\mathcal{X}}\left(y_{2}^{i}, x^{a}, x^{b}, \tau_{1}, \tau_{2}\right) \leq \\
\max \left(Q_{Y_{1} \mid Y_{2} X}\left(\tau_{1} \mid y_{2}^{i}, x_{a}^{i}\right)-Q_{Y_{1} \mid Y_{2} X}\left(\tau_{1} \mid y_{2}^{i}, x_{b}^{i-1}\right), Q_{Y_{1} \mid Y_{2} X}\left(\tau_{1} \mid y_{2}^{i}, x_{a}^{i-1}\right)-Q_{Y_{1} \mid Y_{2} X}\left(\tau_{1} \mid y_{2}^{i}, x_{b}^{i}\right)\right)
\end{gathered}
$$

a result that can be proved by minor modification of the argument of the proof of Theorem 1.

3.3. Sparse support. The rank condition (II) requires that there exist a set of instrumental values of $X$

$$
\mathcal{X}=\left\{x^{i}, x^{j}, x^{i-1}, x^{j-1}\right\}
$$

such that

$$
p^{i}\left(x^{i}\right)=p^{j}\left(x^{j}\right)=p^{i-1}\left(x^{i-1}\right)=p^{j-1}\left(x^{j-1}\right)=\tau_{2} .
$$

When $X$ has sparse support, for example when $X$ does not exhibit continuous variation it may not be possible to find such a set $\mathcal{X}$.

In this case, modification of the rank condition (II) can lead to interval identification. Replace condition (II) by the following condition $\left(\mathrm{II}^{\prime \prime}\right)$.

$\mathrm{II}^{\prime \prime}$. Rank condition. There exists a set of distinct instrumental values of $X$ :

$$
\mathcal{X}=\left\{x^{i}, x^{j}, x^{i-1}, x^{j-1}\right\}
$$

such that

$$
p^{i}\left(x^{i}\right) \leq \tau_{2} \quad p^{i-1}\left(x^{i-1}\right) \geq \tau_{2} \quad p^{j}\left(x^{j}\right) \leq \tau_{2} \quad p^{j-1}\left(x^{j-1}\right) \geq \tau_{2}
$$

The other conditions are unchanged and there is the following Theorem.

Theorem 2. Conditions (I), (II'), (III), (IV) and (V) imply the inequality stated in Theorem 1.

A proof is given in the Appendix. When one or more strong inequalities hold in (12) the bounds in the inequality of Theorem 1 may be wider than those obtained when equalities hold throughout. Further, the conditions of Theorem 2 are more restrictive in this case since they require, that the covariation and monotonicity conditions hold over a larger set of values of $\rho_{2}$ than when strict equalities hold throughout in (12).

\section{Concluding Remarks}

The interval identification result obtained here suggests that point nonparametric identification of $\Delta$ is not possible in a nonseparable model.

If $Y_{2}$ is discrete and $h_{2}$ is strictly increasing then $\rho_{2}$ must be discrete, and then, if $h_{2}$ depends on $X$, changes in $X$ change the locations of the points of support. This is not a case of real econometric interest.

The more interesting case considered here is that in which discrete $Y_{2}$ is generated by continuous $\rho_{2}$ which requires that $h_{2}$ is a step function. But in this case, each value of $Y_{2}$ is associated with a interval of values of $\rho_{2}$ and the data generating value in this interval cannot be identified. 
Therefore it is not possible to "control" precisely for variation in $\rho_{2}$ by fixing $Y_{2}$ at some quantile of its conditional distribution given $X$ and considering alternative instrumental values of $X$, as was proposed in Chesher (2002) for the case in which $Y_{2}$ is continuously distributed.

However the monotonicity condition on $Q_{\rho_{1} \mid \rho_{2} X}$ invoked here allows the impact of $\rho_{2}$ on $\rho_{1}$, and thus on the value delivered by $h_{1}$, to be bounded as $\rho_{2}$ varies within the interval associated with the points of support of $Y_{2}$ that are of interest, and this leads to the interval identification result of this paper.

The result for binary endogenous variables presents an interesting puzzle. Under the conditions proposed there can be interval identification of a structural partial difference with respect to a binary endogenous variable, but the interval does not have finite length. What minimal additional restrictions would lead to identification up to an interval of finite length?

\section{REFERENCES}

Becker, Gary S., And Barry R. Chiswick (1966): "Education and the distribution of earnings," American Economic Review, 56, 358-369.

CARD, DAVID (2001): "Estimating the returns to schooling: Progress on some persistent econometric problems," Econometrica, 69, 1127-1160.

CARD, DAVID (1995): "Earnings, ability and schooling revisited," in Research in Labour Economics, Volume 14, ed., S. Polachek. Greenwich, Conn: JAI Press.

Chaudhuri, Probal (1991): "Nonparametric estimation of regression quantiles and their local Bahadur representation," Annals of Statistics, 19, 760-777.

Chaudhuri, Probal, Kjell Doksum and Alexander Samarov (1997): "On average derivative quantile regression," Annals of Statistics, 25, 715-744.

Chesher, Andrew D. (2002): "Instrumental values," Centre for Microdata Methods and Practice Working Paper 17/02.

Chesher, Andrew D. (2003): "Identification in nonseparable models," forthcoming in Econometrica.

CHISWICK, BARRY R. (1974): Income inequality: regional analyses within a human capital framework. New York: Columbia University Press.

Chiswick, Barry R., And JaCOB Mincer (1972): "Time series changes in personal income inequality," Journal of Political Economy, 80, S34-S66.

Kahn, Shakeeb (2001): "Two-stage rank estimation of quantile index models," Journal of Econometrics, 100, 319-355.

Koenker, Roger W., And Gilbert W. Bassett JR. (1978): "Regression quantiles," Econometrica, 46, 33-50.

Koenker, Roger W. And Vasco D'Orey (1987): "Computing regression quantiles," Journal of the Royal Statistical Society, Series C, 36, 383-393.

LEE, SokBAe (2003): "Efficient semiparametric estimation of a partially linear quantile regression model," Econometric Theory, 19, 1-31.

Manski, Charles F. (1988b): Analog estimation methods in econometrics, New York: Chapman and Hall.

Mincer, Jacob (1974): Schooling experience and earnings. New York: Columbia University Press. 


\section{Appendix: Proofs of Theorems 1 And 2}

First, two lemmata employed in the proofs of the theorems are stated and proved. Lemma 1 provides an expression for the distribution function of $Y_{1}$ conditional on $Y_{2}=y_{2}^{m}$ and $X=x$. Lemma 2 places bounds on the associated conditional quantile function. The proofs of Theorems 1 and 2 follow.

\section{Lemma 1}

Under condition (I) and the weak monotonicity (normalised non-decreasing) of $h_{1}$ with respect to $\rho_{1}$ required by condition (III), the conditional distribution function of $Y_{1}$ given $Y_{2}=y_{2}^{m}$ and $X=x$ is as follows.

$$
F_{Y_{1} \mid Y_{2} X}\left(y_{1} \mid y_{2}^{m}, x\right)=\frac{1}{p_{m}(x)} \int_{p^{m-1}(x)}^{p^{m}(x)} F_{\rho_{1} \mid \rho_{2} X}\left(\sup _{p}\left\{p: h_{1}\left(y_{2}^{m}, x, p\right) \leq y_{1}\right\} \mid \rho_{2}, x\right) d \rho_{2}
$$

If $h_{1}$ is strictly monotonic (normalised increasing) with respect to $\rho_{1}$ then

$$
F_{Y_{1} \mid Y_{2} X}\left(y_{1} \mid y_{2}^{m}, x\right)=\frac{1}{p_{m}(x)} \int_{p^{m-1}(x)}^{p^{m}(x)} F_{\rho_{1} \mid \rho_{2} X}\left(h_{1}^{-1}\left(y_{2}^{m}, x, y_{1}\right) \mid \rho_{2}, x\right) d \rho_{2}
$$

where $h_{1}^{-1}\left(y_{2}^{m}, x, y_{1}\right)$ satisfies

$$
y_{1}=h_{1}\left(y_{2}^{m}, x, h_{1}^{-1}\left(y_{2}^{m}, x, y_{1}\right)\right) .
$$

\section{Proof of Lemma 1}

The conditional distribution function of $Y_{1}$ given $Y_{2}=y_{2}^{m}$ and $X=x$ is defined as follows.

$$
F_{Y_{1} \mid Y_{2} X}\left(y_{1} \mid y_{2}^{m}, x\right) \equiv \frac{P\left[Y_{1} \leq y_{1} \cap Y_{2}=y_{2}^{m} \mid X=x\right]}{P\left[Y_{2}=y_{2}^{m} \mid X=x\right]}
$$

Given $X=x$, in terms of events, since $h_{1}$ is a non-decreasing function of $\rho_{1}$,

$$
\left\{Y_{1} \leq y_{1} \cap Y_{2}=y_{2}^{m}\right\}=\left\{\rho_{1} \leq \sup _{p}\left\{p: h_{1}\left(y_{2}^{m}, x, p\right) \leq y_{1}\right\} \cap\left\{p^{m-1}(x)<\rho_{2} \leq p^{m}(x)\right\}\right.
$$

and so

$$
P\left[Y_{1} \leq y_{1} \cap Y_{2}=y_{2}^{m} \mid X=x\right]=\int_{p^{m-1}(x)}^{p^{m}(x)} F_{\rho_{1} \mid \rho_{2} X}\left(\sup _{p}\left\{p: h_{1}\left(y_{2}^{m}, x, p\right) \leq y_{1}\right\} \mid \rho_{2}, x\right) d \rho_{2}
$$

and the first result of the Lemma follows on dividing by $P\left[Y_{2}=y_{2}^{m} \mid X=x\right]=p_{m}(x)$.

If $h_{1}$ is an increasing function of $\rho_{1}$ then

$$
\sup _{p}\left\{p: h_{1}\left(y_{2}^{m}, x, p\right) \leq y_{1}\right\}=h_{1}^{-1}\left(y_{2}^{m}, x, y_{1}\right)
$$

and substituting in (A2) leads to the second result of the Lemma.

The conditional $\tau_{1}$-quantile of $Y_{1}$ given $Y_{2}=y_{2}^{m}$ and $X=x$ is

$$
Q_{Y_{1} \mid Y_{2} X}\left(\tau_{1} \mid y_{2}^{m}, x\right) \equiv \inf _{q}\left\{q: \frac{1}{p_{m}(x)} \int_{p^{m-1}(x)}^{p^{m}(x)} F_{\rho_{1} \mid \rho_{2} X}\left(\sup _{p}\left\{p: h_{1}\left(y_{2}^{m}, x, p\right) \leq q\right\} \mid \rho_{2}, x\right) d \rho_{2} \geq \tau_{1}\right\}
$$


and when $h_{1}$ is increasing with respect to $\rho_{1}$ :

$$
Q_{Y_{1} \mid Y_{2} X}\left(\tau_{1} \mid y_{2}^{m}, x\right) \equiv \inf _{q}\left\{q: \frac{1}{p_{m}(x)} \int_{p^{m-1}(x)}^{p^{m}(x)} F_{\rho_{1} \mid \rho_{2} X}\left(h_{1}^{-1}\left(y_{2}^{m}, x, q\right) \mid \rho_{2}, x\right) d \rho_{2} \geq \tau_{1}\right\} .
$$

Lemma 2 places bounds on this conditional quantile.

\section{Lemma 2} then

Under conditions (I) and (III), if $F_{\rho_{1} \mid \rho_{2} X}$ is non-decreasing in $\rho_{2}$ for $\rho_{2} \in\left[p^{m-1}(x), p^{m}(x)\right]$

$h_{1}\left(y_{2}^{m}, x, Q_{\rho_{1} \mid \rho_{2} X}\left(\tau_{1} \mid p^{m}(x), x\right)\right) \leq Q_{Y_{1} \mid Y_{2} X}\left(\tau_{1} \mid y_{2}^{m}, x\right) \leq h_{1}\left(y_{2}^{m}, x, Q_{\rho_{1} \mid \rho_{2} X}\left(\tau_{1} \mid p^{m-1}(x), x\right)\right)$

with the inequalities reversed if $F_{\rho_{1} \mid \rho_{2} X}$ is non-increasing in $\rho_{2}$ for $\rho_{2} \in\left[p^{m-1}(x), p^{m}(x)\right]$.

\section{Proof of Lemma 2}

If $F_{\rho_{1} \mid \rho_{2} X}$ is non-decreasing in $\rho_{2}$ for $\rho_{2} \in\left[p^{m-1}(x), p^{m}(x)\right]$ then, recalling the result of Lemma 1, replacing $\rho_{2}$ in the integrand first by $p^{m-1}(x)$ and then by $p^{m}(x)$ yields:

$$
\begin{gathered}
\frac{1}{p_{m}(x)} \int_{p^{m-1}(x)}^{p^{m}(x)} F_{\rho_{1} \mid \rho_{2} X}\left(\sup _{p}\left\{p: h_{1}\left(y_{2}^{m}, x, p\right) \leq y_{1}\right\} \mid p^{m-1}(x), x\right) d \rho_{2} \\
\leq F_{Y_{1} \mid Y_{2} X}\left(y_{1} \mid y_{2}^{m}, x\right) \leq \\
\frac{1}{p_{m}(x)} \int_{p^{m-1}(x)}^{p^{m}(x)} F_{\rho_{1} \mid \rho_{2} X}\left(\sup _{p}\left\{p: h_{1}\left(y_{2}^{m}, x, p\right) \leq y_{1}\right\} \mid p^{m}(x), x\right) d \rho_{2}
\end{gathered}
$$

and since, for example

$$
\begin{array}{r}
\frac{1}{p_{m}(x)} \int_{p^{m-1}(x)}^{p^{m}(x)} F_{\rho_{1} \mid \rho_{2} X}\left(\sup _{p}\left\{p: h_{1}\left(y_{2}^{m}, x, p\right) \leq y_{1}\right\} \mid p^{m}(x), x\right) d \rho_{2} \\
=F_{\rho_{1} \mid \rho_{2} X}\left(\sup _{p}\left\{p: h_{1}\left(y_{2}^{m}, x, p\right) \leq y_{1}\right\} \mid p^{m}(x), x\right)
\end{array}
$$

there is, for all $y_{1}$, the following.

$$
\begin{gathered}
F_{\rho_{1} \mid \rho_{2} X}\left(\sup _{p}\left\{p: h_{1}\left(y_{2}^{m}, x, p\right) \leq y_{1}\right\} \mid p^{m-1}(x), x\right) \\
\leq F_{Y_{1} \mid Y_{2} X}\left(y_{1} \mid y_{2}^{m}, x\right) \leq \\
F_{\rho_{1} \mid \rho_{2} X}\left(\sup _{p}\left\{p: h_{1}\left(y_{2}^{m}, x, p\right) \leq y_{1}\right\} \mid p^{m}(x), x\right)
\end{gathered}
$$

If $h_{1}$ is an increasing function of $\rho_{1}$ then in $(\mathrm{A} 4) \sup _{p}\left\{p: h_{1}\left(y_{2}^{m}, x, p\right) \leq y_{1}\right\}$ can be replaced by $h_{1}^{-1}\left(y_{2}^{m}, x, y_{1}\right)$.

If $F_{\rho_{1} \mid \rho_{2} X}$ is non-increasing in $\rho_{2}$ for $\rho_{2} \in\left[p^{m-1}(x), p^{m}(x)\right]$ the inequality (A4) is reversed. ${ }^{12}$

\footnotetext{
${ }^{12}$ Monotonicity of $F_{\rho_{1} \mid \rho_{2} X}$ with respect to $\rho_{2}$ is a stronger condition than required but it has the advantage that it is easily interpreted. The inequality (A4) holds if for all $\rho_{2} \in\left[p^{m-1}(x), p^{m}(x)\right]$,

$$
\begin{aligned}
& F_{\rho_{1} \mid \rho_{2} X}\left(\sup _{p}\left\{p: h_{1}\left(y_{2}^{m}, x, p\right) \leq y_{1}\right\} \mid \rho_{2}, x\right) \in\left[\min \left(A_{m}^{m-1}, A_{m}^{m}\right), \max \left(A_{m}^{m-1}, A_{m}^{m}\right)\right] \\
& A_{m}^{m-1} \equiv F_{\rho_{1} \mid \rho_{2} X}\left(\sup _{p}\left\{p: h_{1}\left(y_{2}^{m}, x, p\right) \leq y_{1}\right\} \mid p^{m-1}(x), x\right) \\
& A_{m}^{m}\left.\equiv F_{\rho_{1} \mid \rho_{2} X}\left(\sup _{p}\left\{p: h_{1}\left(y_{2}^{m}, x, p\right) \leq y_{1}\right\} \mid p^{m}(x), x\right)\right]
\end{aligned}
$$
}

for which the assumed monotonicity condition is sufficient. 
Since the functions on the left and the right of the inequality (A4) bound $F_{Y_{1} \mid Y_{2} X}\left(y_{1} \mid y_{2}^{m}, x\right)$ respectively below and above, the solutions, $q_{m-1}$ and $q_{m}$ to

$$
\begin{aligned}
q_{m-1} & =\inf _{q}\left\{q: F_{\rho_{1} \mid \rho_{2} X}\left(\sup _{p}\left\{p: h_{1}\left(y_{2}^{m}, x, p\right) \leq q\right\} \mid p^{m-1}(x), x\right) \geq \tau_{1}\right\} \\
q_{m} & =\inf _{q}\left\{q: F_{\rho_{1} \mid \rho_{2} X}\left(\sup _{p}\left\{p: h_{1}\left(y_{2}^{m}, x, p\right) \leq q\right\} \mid p^{m}(x), x\right) \geq \tau_{1}\right\}
\end{aligned}
$$

bound

$$
\inf _{q}\left\{q: F_{Y_{1} \mid Y_{2} X}\left(q \mid y_{2}^{m}, x\right) \geq \tau_{1}\right\} \equiv Q_{Y_{1} \mid Y_{2} X}\left(\tau_{1} \mid y_{2}^{m}, x\right)
$$

respectively above and below, that is:

$$
q_{m} \leq Q_{Y_{1} \mid Y_{2} X}\left(\tau_{1} \mid y_{2}^{m}, x\right) \leq q_{m-1} .
$$

To aid understanding, consider Figure 1 where the distribution function $F_{Y_{1} \mid Y_{2} X}\left(y_{1} \mid y_{2}^{m}, x\right)$ is the solid cadlag step function and the upper $(\mathrm{u})$ and lower (l) bounding functions in (A4) are drawn respectively dashed and dotted. A value of $\tau_{1}$ is shown as a solid horizontal line. In the example drawn, $q_{m}<q<q_{m-1}$. If $\tau_{1}$ was slightly smaller then $q$ and $q_{m-1}$ would coincide, and a further small decrease would result in coincidence of $q_{m}$ and $q$.

The relationships between the values $q_{m-1}$ and $q_{m}$ and the structural function $h_{1}$ are now determined.

Consider $q_{m}$. For any choice of $q$,

$$
F_{\rho_{1} \mid \rho_{2} X}\left(\sup _{p}\left\{p: h_{1}\left(y_{2}^{m}, x, p\right) \leq q\right\} \mid p^{m}(x), x\right) \geq \tau_{1}
$$

if and only if

$$
Q_{\rho_{1} \mid \rho_{2} X}\left(\tau_{1} \mid p^{m}(x), x\right) \leq \sup _{p}\left\{p: h_{1}\left(y_{2}^{m}, x, p\right) \leq q\right\}
$$

because $F_{\rho_{1} \mid \rho_{2} X}$ is strictly increasing in its first argument; recall $\rho_{1}$ is required to be continuously distributed. Therefore

$$
q_{m}=\inf _{q}\left\{q: Q_{\rho_{1} \mid \rho_{2} X}\left(\tau_{1} \mid p^{m}(x), x\right) \leq \sup _{p}\left\{p: h_{1}\left(y_{2}^{m}, x, p\right) \leq q\right\}\right\} .
$$

Consider

$$
q^{*}=h_{1}\left(y_{2}^{m}, x, Q_{\rho_{1} \mid \rho_{2} X}\left(\tau_{1} \mid p^{m}(x), x\right)\right)
$$

which is a candidate value for $q_{m}$ because,

$$
Q_{\rho_{1} \mid \rho_{2} X}\left(\tau_{1} \mid p^{m}(x), x\right) \leq \sup _{p}\left\{p: h_{1}\left(y_{2}^{m}, x, p\right) \leq q^{*}\right\} .
$$

This holds as an equality when $h_{1}$ is a strictly increasing function of $\rho_{1}$ - this follows directly from equation (A3) and the definition, (A1), of the inverse function $h_{1}^{-1}\left(y_{2}^{m}, x, y_{1}\right)$ - and in that case,

$$
q_{m} \equiv \inf _{q}\left\{q: Q_{\rho_{1} \mid \rho_{2} X}\left(\tau_{1} \mid p^{m}(x), x\right) \leq h_{1}^{-1}\left(y_{2}^{m}, x, q\right)\right\}=q^{*} .
$$

The case in which $h_{1}$ is a non-decreasing caglad step function of $\rho_{1}$ is drawn in Figure 2. Ends of steps at which $h_{1}$ is continuous from the left are drawn as filled circles. The graph shows a value of $Q_{\rho_{1} \mid \rho_{2} X}\left(\tau_{1} \mid p^{m}(x), x\right)$ and the candidate value $q^{*}$. The value of

$$
p^{*} \equiv \sup _{p}\left\{p: h_{1}\left(y_{2}^{m}, x, p\right) \leq q^{*}\right\}
$$


is indicated in Figure 2 and clearly $p^{*} \geq Q_{\rho_{1} \mid \rho_{2} X}\left(\tau_{1} \mid p^{m}(x), x\right)$, demonstrating that the inequality (A5) does hold. that

It is evident from Figure 2 that for $q<q^{*}$ there is no $p \geq Q_{\rho_{1} \mid \rho_{2} X}\left(\tau_{1} \mid p^{m}(x), x\right)$ such

$$
Q_{\rho_{1} \mid \rho_{2} X}\left(\tau_{1} \mid p^{m}(x), x\right) \leq \sup _{p}\left\{p: h_{1}\left(y_{2}^{m}, x, p\right) \leq q\right\}
$$

and so $q_{m}=q^{*}$.

A similar argument shows that $q_{m-1}$ is as follows.

$$
q_{m-1}=h_{1}\left(y_{2}^{m}, x, Q_{\rho_{1} \mid \rho_{2} X}\left(\tau_{1} \mid p^{m-1}(x), x\right)\right)
$$

Therefore, in the case in which $F_{\rho_{1} \mid \rho_{2} X}$ is non-decreasing in $\rho_{2}$ for $\rho_{2} \in\left[p^{m-1}(x), p^{m}(x)\right]$, there is the following inequality.

$h_{1}\left(y_{2}^{m}, x, Q_{\rho_{1} \mid \rho_{2} X}\left(\tau_{1} \mid p^{m}(x), x\right)\right) \leq Q_{Y_{1} \mid Y_{2} X}\left(\tau_{1} \mid y_{2}^{m}, x\right) \leq h_{1}\left(y_{2}^{m}, x, Q_{\rho_{1} \mid \rho_{2} X}\left(\tau_{1} \mid p^{m-1}(x), x\right)\right)$

A similar argument for the case in which $F_{\rho_{1} \mid \rho_{2} X}$ is non-increasing in $\rho_{2}$ for $\rho_{2} \in$ $\left[p^{m-1}(x), p^{m}(x)\right]$ produces the reverse of this inequality

$h_{1}\left(y_{2}^{m}, x, Q_{\rho_{1} \mid \rho_{2} X}\left(\tau_{1} \mid p^{m-1}(x), x\right)\right) \leq Q_{Y_{1} \mid Y_{2} X}\left(\tau_{1} \mid y_{2}^{m}, x\right) \leq h_{1}\left(y_{2}^{m}, x, Q_{\rho_{1} \mid \rho_{2} X}\left(\tau_{1} \mid p^{m}(x), x\right)\right)$

and combining the two preceding inequalities gives the result of the Lemma.

\section{Proof of Theorem 1}

Consider the case in which, for $x \in \mathcal{X}, Q_{\rho_{1} \mid \rho_{2} X}$ is non-increasing in $\rho_{2}$ over the intervals $\mathcal{R}_{2}(x)$ defined in condition (III). Since $F_{\rho_{1} \mid \rho_{2} X}$ is the inverse function of $Q_{\rho_{1} \mid \rho_{2} X}$ this implies that $F_{\rho_{1} \mid \rho_{2} X}$ is non-decreasing over the intervals $\mathcal{R}_{2}(x)$ defined in condition (III). In the inequality of Lemma 2 set $m=i$, and set $x=x^{i}$ giving

$$
h_{1}\left(y_{2}^{i}, x^{i}, Q_{\rho_{1} \mid \rho_{2} X}\left(\tau_{1} \mid p^{i}\left(x^{i}\right), x^{i}\right)\right) \leq Q_{Y_{1} \mid Y_{2} X}\left(\tau_{1} \mid y_{2}^{i}, x^{i}\right)
$$

and set $m=j$ and $x=x^{j-1}$ giving

$$
Q_{Y_{1} \mid Y_{2} X}\left(\tau_{1} \mid y_{2}^{j}, x^{j-1}\right) \leq h_{1}\left(y_{2}^{j}, x^{j-1}, Q_{\rho_{1} \mid \rho_{2} X}\left(\tau_{1} \mid p^{j-1}\left(x^{j-1}\right), x^{j-1}\right)\right) .
$$

Invoking the rank and order conditions (II and V) these inequalities simplify to

$$
\begin{gathered}
h_{1}\left(y_{2}^{i}, x, Q_{\rho_{1} \mid \rho_{2} X}\left(\tau_{1} \mid \tau_{2}, x^{i}\right)\right) \leq Q_{Y_{1} \mid Y_{2} X}\left(\tau_{1} \mid y_{2}^{i}, x^{i}\right) \\
Q_{Y_{1} \mid Y_{2} X}\left(\tau_{1} \mid y_{2}^{j}, x^{j-1}\right) \leq h_{1}\left(y_{2}^{j}, x, Q_{\rho_{1} \mid \rho_{2} X}\left(\tau_{1} \mid \tau_{2}, x^{j-1}\right)\right)
\end{gathered}
$$

where $x$ is any member of $\mathcal{X}$, the set of instrumental values and $h_{1}$ is insensitive through its $x$ argument to choice of $x \in \mathcal{X}$.

The covariation condition (IV) implies that the conditional quantiles of $\rho_{1}$ that appear in the two inequalities take the same value, namely $r_{1}$. Thus for $x \in \mathcal{X}$ there is the following.

$$
\begin{gathered}
h_{1}\left(y_{2}^{i}, x, r_{1}\right) \leq Q_{Y_{1} \mid Y_{2} X}\left(\tau_{1} \mid y_{2}^{i}, x^{i}\right) \\
Q_{Y_{1} \mid Y_{2} X}\left(\tau_{1} \mid y_{2}^{j}, x^{j-1}\right) \leq h_{1}\left(y_{2}^{j}, x, r_{1}\right)
\end{gathered}
$$

Adding the two inequalities and rearranging there is, for $x \in \mathcal{X}$ :

$$
h_{1}\left(y_{2}^{j}, x, r_{1}\right)-h_{1}\left(y_{2}^{i}, x, r_{1}\right) \geq Q_{Y_{1} \mid Y_{2} X}\left(\tau_{1} \mid y_{2}^{j}, x^{j-1}\right)-Q_{Y_{1} \mid Y_{2} X}\left(\tau_{1} \mid y_{2}^{i}, x^{i}\right) .
$$

Setting $m=j$ and $x=x^{j}$ and then $m=i$ and $x=x^{i-1}$ in the inequality of Lemma 2 , and arguing along similar lines yields the following inequality.

$$
Q_{Y_{1} \mid Y_{2} X}\left(\tau_{1} \mid y_{2}^{j}, x^{j}\right)-Q_{Y_{1} \mid Y_{2} X}\left(\tau_{1} \mid y_{2}^{i}, x^{i-1}\right) \geq h_{1}\left(y_{2}^{j}, x, r_{1}\right)-h_{1}\left(y_{2}^{i}, x, r_{1}\right)
$$


Combining (A6) and (A7) gives the following for $x \in \mathcal{X}$.

$$
\begin{gathered}
Q_{Y_{1} \mid Y_{2} X}\left(\tau_{1} \mid y_{2}^{j}, x^{j-1}\right)-Q_{Y_{1} \mid Y_{2} X}\left(\tau_{1} \mid y_{2}^{i}, x^{i}\right) \\
\leq h_{1}\left(y_{2}^{j}, x, r_{1}\right)-h_{1}\left(y_{2}^{i}, x, r_{1}\right) \leq \\
Q_{Y_{1} \mid Y_{2} X}\left(\tau_{1} \mid y_{2}^{j}, x^{j}\right)-Q_{Y_{1} \mid Y_{2} X}\left(\tau_{1} \mid y_{2}^{i}, x^{i-1}\right)
\end{gathered}
$$

When, for $x \in \mathcal{X}, Q_{\rho_{1} \mid \rho_{2} X}$ is non-decreasing in $\rho_{2}$ over the intervals $\mathcal{R}_{2}(x)$ defined in condition (III) (which implies $F_{\rho_{1} \mid \rho_{2} X}$ is non-increasing in $\rho_{2}$ over these intervals) these inequalities are reversed and combining the two sets of inequalities gives the result stated in the theorem.

\section{Proof of Theorem 2}

As in the proof of Theorem 1, first consider the case in which, for $x \in \mathcal{X}, Q_{\rho_{1} \mid \rho_{2} X}$ is non-increasing in $\rho_{2}$ over the intervals $\mathcal{R}_{2}(x)$ defined in condition (III). Since $F_{\rho_{1} \mid \rho_{2} X}$ is the inverse function of $Q_{\rho_{1} \mid \rho_{2} X}$ this implies that $F_{\rho_{1} \mid \rho_{2} X}$ is non-decreasing over the intervals $\mathcal{R}_{2}(x)$ defined in condition (III).

Recall that in Theorem 2 the instrumental values satisfy the following weak inequalities.

$$
\begin{array}{ll}
p^{i}\left(x^{i}\right) \leq \tau_{2} & p^{i-1}\left(x^{i-1}\right) \geq \tau_{2} \\
p^{j}\left(x^{j}\right) \leq \tau_{2} & p^{j-1}\left(x^{j-1}\right) \geq \tau_{2}
\end{array}
$$

Lemma 2 implies

$$
\begin{aligned}
h_{1}\left(y_{2}^{i}, x^{i}, Q_{\rho_{1} \mid \rho_{2} X}\left(\tau_{1} \mid p^{i}\left(x^{i}\right), x^{i}\right)\right) & \leq Q_{Y_{1} \mid Y_{2} X}\left(\tau_{1} \mid y_{2}^{i}, x^{i}\right) \\
Q_{Y_{1} \mid Y_{2} X}\left(\tau_{1} \mid y_{2}^{j}, x^{j-1}\right) & \leq h_{1}\left(y_{2}^{j}, x^{j-1}, Q_{\rho_{1} \mid \rho_{2} X}\left(\tau_{1} \mid p^{j-1}\left(x^{j-1}\right), x^{j-1}\right)\right)
\end{aligned}
$$

and since $Q_{\rho_{1} \mid \rho_{2} X}$ is a non-increasing function of $\rho_{2}$ (recall $h_{1}$ is normalised non-decreasing in its final argument) there is the following pair of inequalities.

$$
\begin{aligned}
h_{1}\left(y_{2}^{i}, x^{i}, Q_{\rho_{1} \mid \rho_{2} X}\left(\tau_{1} \mid \tau_{2}, x^{i}\right)\right) & \leq h_{1}\left(y_{2}^{i}, x^{i}, Q_{\rho_{1} \mid \rho_{2} X}\left(\tau_{1} \mid p^{i}\left(x^{i}\right), x^{i}\right)\right) \\
h_{1}\left(y_{2}^{j}, x^{j-1}, Q_{\rho_{1} \mid \rho_{2} X}\left(\tau_{1} \mid p^{j-1}\left(x^{j-1}\right), x^{j-1}\right)\right) & \leq h_{1}\left(y_{2}^{j}, x^{j-1}, Q_{\rho_{1} \mid \rho_{2} X}\left(\tau_{1} \mid \tau_{2}, x^{j-1}\right)\right)
\end{aligned}
$$

Combining the preceding two pairs of inequalities yields

$$
\begin{aligned}
h_{1}\left(y_{2}^{i}, x^{i}, Q_{\rho_{1} \mid \rho_{2} X}\left(\tau_{1} \mid \tau_{2}, x^{i}\right)\right) & \leq Q_{Y_{1} \mid Y_{2} X}\left(\tau_{1} \mid y_{2}^{i}, x^{i}\right) \\
Q_{Y_{1} \mid Y_{2} X}\left(\tau_{1} \mid y_{2}^{j}, x^{j-1}\right) & \leq h_{1}\left(y_{2}^{j}, x^{j-1}, Q_{\rho_{1} \mid \rho_{2} X}\left(\tau_{1} \mid \tau_{2}, x^{j-1}\right)\right)
\end{aligned}
$$

and adding these inequalities and rearranging there is the following.

$$
\begin{aligned}
& Q_{Y_{1} \mid Y_{2} X}\left(\tau_{1} \mid y_{2}^{j}, x^{j-1}\right)-Q_{Y_{1} \mid Y_{2} X}\left(\tau_{1} \mid y_{2}^{i}, x^{i}\right) \\
& \quad \leq h_{1}\left(y_{2}^{j}, x^{j-1}, Q_{\rho_{1} \mid \rho_{2} X}\left(\tau_{1} \mid \tau_{2}, x^{j-1}\right)\right)-h_{1}\left(y_{2}^{i}, x^{i}, Q_{\rho_{1} \mid \rho_{2} X}\left(\tau_{1} \mid \tau_{2}, x^{i}\right)\right)
\end{aligned}
$$

Setting $m=j$ and $x=x^{j}$ and then $m=i$ and $x=x^{i-1}$ in the inequality of Lemma 2 and arguing as above yields the following inequality.

$$
\begin{gathered}
h_{1}\left(y_{2}^{j}, x^{j}, Q_{\rho_{1} \mid \rho_{2} X}\left(\tau_{1} \mid \tau_{2}, x^{j}\right)\right)-h_{1}\left(y_{2}^{i}, x^{i-1}, Q_{\rho_{1} \mid \rho_{2} X}\left(\tau_{1} \mid \tau_{2}, x^{i-1}\right)\right) \\
\leq Q_{Y_{1} \mid Y_{2} X}\left(\tau_{1} \mid y_{2}^{j}, x^{j}\right)-Q_{Y_{1} \mid Y_{2} X}\left(\tau_{1} \mid y_{2}^{i}, x^{i-1}\right) .
\end{gathered}
$$

Combining the last pair of inequalities and invoking the order and covariation restrictions and using the definition of $r_{1}$, there is, for $x \in \mathcal{X}$, the following inequality.

$$
Q_{Y_{1} \mid Y_{2} X}\left(\tau_{1} \mid y_{2}^{j}, x^{j-1}\right)-Q_{Y_{1} \mid Y_{2} X}\left(\tau_{1} \mid y_{2}^{i}, x^{i}\right)
$$




$$
\begin{gathered}
\leq h_{1}\left(y_{2}^{j}, x, r_{1}\right)-h_{1}\left(y_{2}^{i}, x, r_{1}\right) \leq \\
Q_{Y_{1} \mid Y_{2} X}\left(\tau_{1} \mid y_{2}^{j}, x^{j}\right)-Q_{Y_{1} \mid Y_{2} X}\left(\tau_{1} \mid y_{2}^{i}, x^{i-1}\right) .
\end{gathered}
$$

In the case in which, for $x \in \mathcal{X}, F_{\rho_{1} \mid \rho_{2} X}$ is a non-increasing function of $\rho_{2}$ for $\rho_{2} \in$ $R_{2}(x)$, which implies that $Q_{\rho_{1} \mid \rho_{2} X}$ is a non-decreasing function of $\rho_{2}$ for $\rho_{2} \in R_{2}(x)$, applying the same argument leads to the reverse of the preceding inequality. Combining the results yields the inequality as stated in Theorem 1 and thus the required result. 


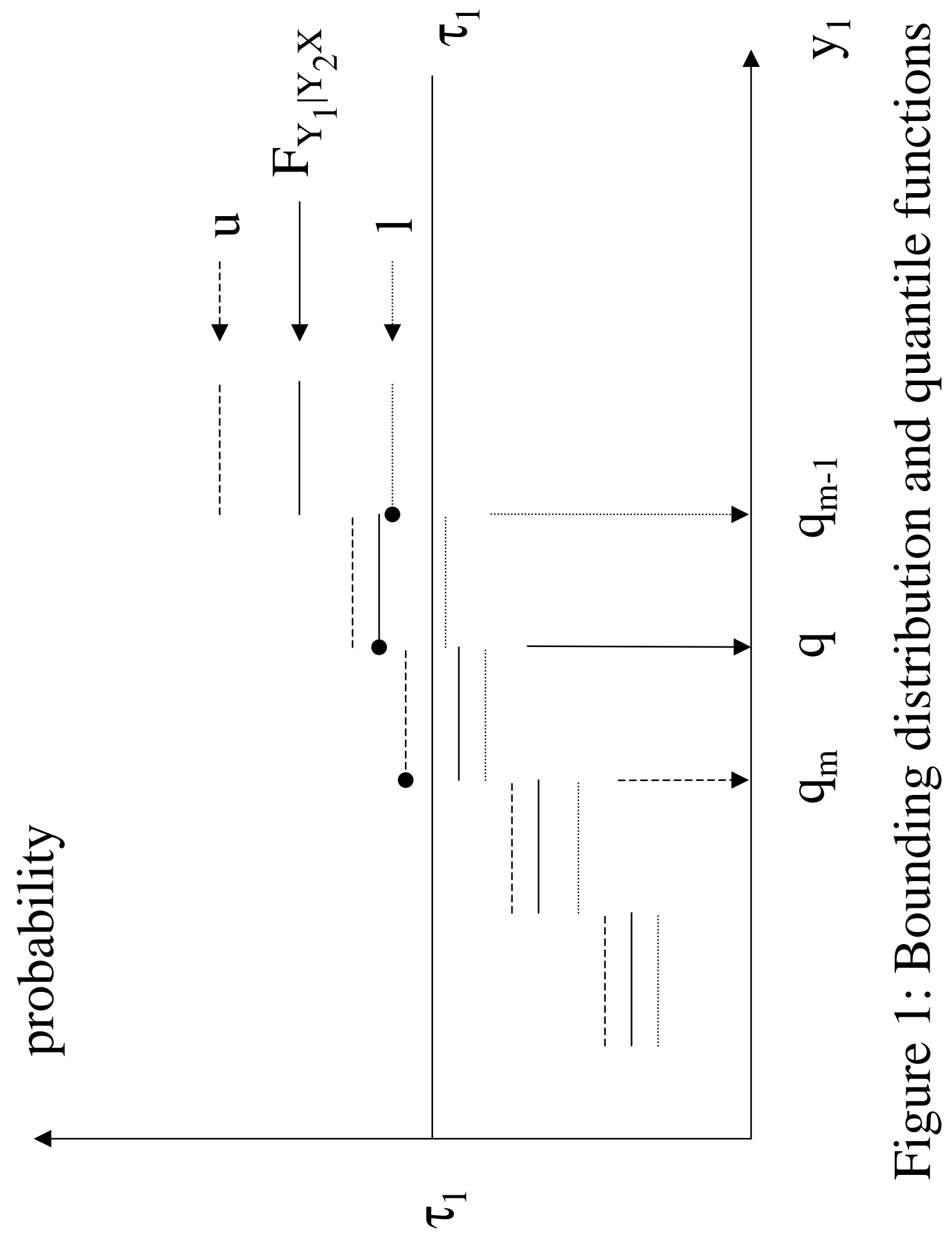




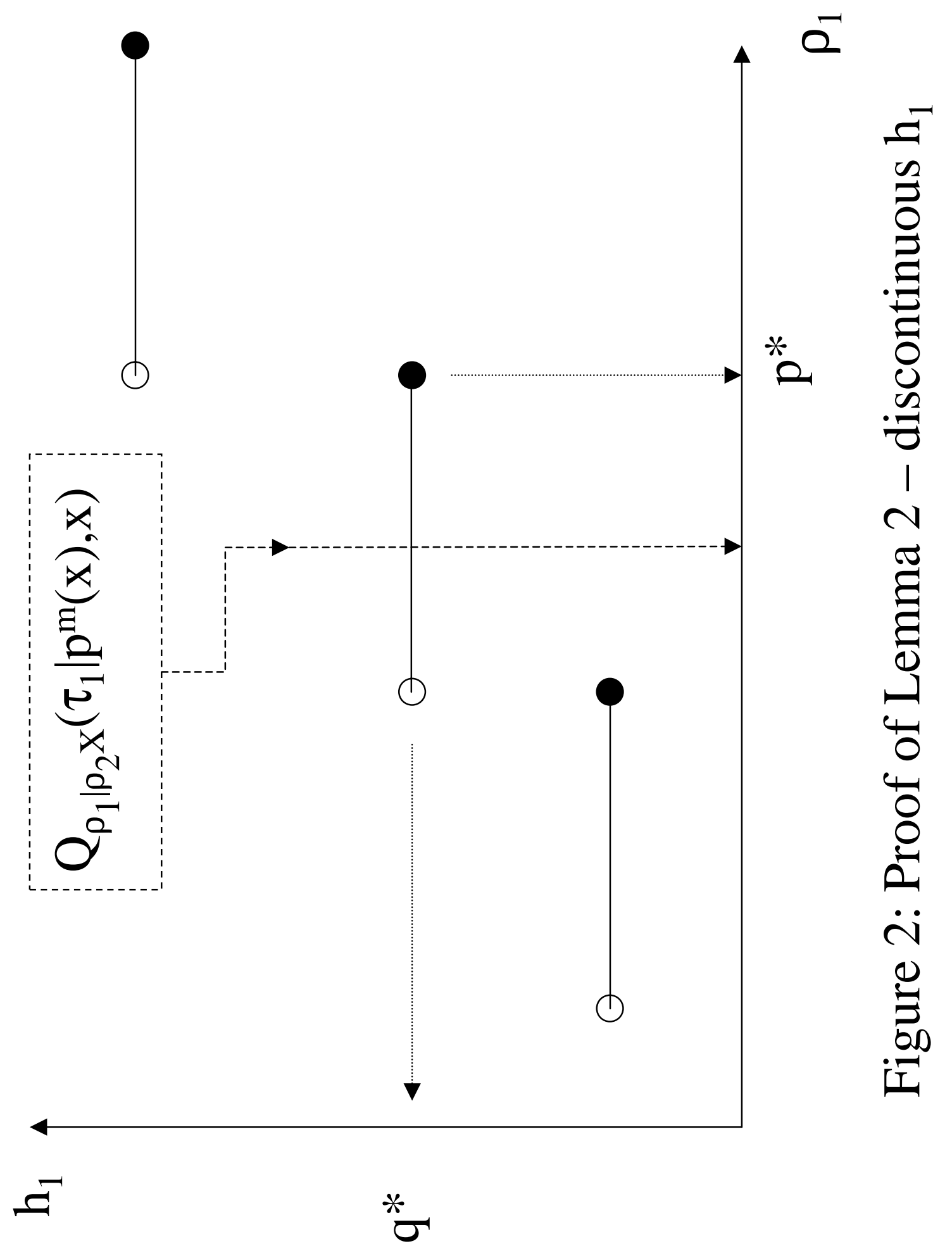

